\title{
Kisspeptin inhibits cancer growth and metastasis via activation of EIF2AK2
}

\author{
TAE-HUN KIM and SUNG-GOOK CHO \\ Department of Biotechnology, Korea National University of Transportation, \\ Jeungpyong, Chungbuk 368-701, Republic of Korea
}

Received October 11, 2016; Accepted March 24, 2017

DOI: $10.3892 / \mathrm{mmr} .2017 .7578$

\begin{abstract}
Kisspeptin is a protein encoded by the KISSI gene, which has been reported to suppress the metastatic capabilities of various types of cancer cells, through the activation of its G-protein coupled receptor GPR54. However, the molecular mechanisms underlying the involvement of kisspeptin-mediated signaling in the inhibition of cancer cell migration and invasion have yet to be elucidated. The present in vitro cell proliferation, migration and invasion assays and in vivo experimental metastasis studies demonstrated that kisspeptin-induced eukaryotic translation initiation factor $2 \alpha$ kinase 2 (EIF2AK2) activation suppressed the metastatic capabilities of several types of cancer cells. Kisspeptin was revealed to inhibit the migratory and invasive abilities of highly metastatic breast SK-BR-3, prostatic PC-3 and colorectal adenocarcinoma LoVo human cancer cell lines, whereas its inhibitory effects were abolished following the silencing of EIF2AK2 expression using RNA interference. Similarly, kisspeptin failed to inhibit the migration and invasion of mouse embryonic fibroblasts following the deletion of the EIF2AK2 gene. Furthermore, kisspeptin was demonstrated to activate Ras homolog gene family member A (RhoA)-dependent signaling, and to phosphorylate EIF2AK2 via RhoA-mediated pathways in various cancer cells. In addition, results obtained from nude mice bearing LoVo-derived xenograft tumors revealed that kisspeptin inhibited tumor growth through an EIF2AK2-dependent mechanism, and an in vivo metastasis assay identified kisspeptin-activated EIF2AK2 signaling as critical for the suppression of distant metastasis. The present study concluded that kisspeptin represses cancer metastasis via EIF2AK2 signaling, thus clarifying the role of kisspeptin signaling in complicated cancer metastasis signaling network.
\end{abstract}

Correspondence to: Dr Sung-Gook Cho, Department of Biotechnology, Korea National University of Transportation, 61 University Road, Jeungpyong, Chungbuk 368-701, Republic of Korea

E-mail: chosg@ut.ac.kr

Key words: kisspeptin, KISS1, G-protein coupled receptor 54, eukaryotic translation initiation factor $2 \alpha$ kinase 2 , cancer
Therefore, kisspeptin treatment may be a choice for blocking metastases.

\section{Introduction}

Kisspeptin is a protein encoded by the KISS1 gene in humans, which has been reported to serve a diverse role in various physiological and pathological conditions (1-5). Kisspeptin has been reported to suppress the metastatic capabilities of various types of cancer cells, through the activation of its G-protein coupled receptor (GPR) 54 (5-7). Kisspeptin has been demonstrated to activate various cytosolic protein kinases, including $\mathrm{B}, \mathrm{C}$ and D in several types of cells in vitro (8-10). However, kisspeptin-mediated signaling pathways are complex, and the molecular mechanisms underlying the involvement of kisspeptin-induced intracellular signaling in the regulation of the migratory and invasive capabilities of cancer cells have yet to be fully elucidated $(9,11-13)$.

The eukaryotic translation initiation factor $2 \alpha$ kinase 2 (EIF2AK2; also known as double-stranded RNA-activated protein kinase, PKR), has been revealed to be ubiquitously expressed across numerous cell types, and may be activated under various types of cellular stress, including viral infections, hypoxia and nutritional depletion (14). EIF2AK2 activation has been reported to suppress tumor growth (15-18); however, its expression and activity levels have been demonstrated to be increased in various types of cancer cells, including breast, lung and colorectal cancer cells (19-21). It has previously been reported that invasive ductal carcinoma cells exhibit increased EIF2AK2 expression levels compared with adjacent normal breast tissue (21); however, the expression and activity levels of EIF2AK2 have been negatively correlated with the metastatic capabilities of breast cancer cells (22). Furthermore, the double-stranded RNA-mediated activation of EIF2AK2 has been implicated in the regulation of breast cancer cell motility (23). These results suggest that EIF2AK2 activity may be critical for the suppression of cancer metastasis. Conversely, EIF2AK2 has been reported to be implicated in hepatocellular carcinoma growth and migration, as well as gastric cancer metastasis $(24,25)$. Therefore, it may be hypothesized that its anti-tumor or oncogenic role is determined by the type of cancer cell.

Previous studies have proposed a role for EIF2AK2 in the regulation of cytoskeletal dynamics $(23,26)$, which appear to 
be similar to the roles of kisspeptin in the regulation of cellular architecture $(7,8,13)$. However, whether kisspeptin may inhibit cancer cell migration and invasion through the modulation of EIF2AK2 has yet to be elucidated. Therefore, the present study aimed to investigate the role of EIF2AK2 in the mechanisms underlying the inhibitory effects of kisspeptin on cancer metastasis. The results of the present study demonstrated that kisspeptin activated EIF2AK2 in several highly metastatic types of cancer cells. Furthermore, kisspeptin failed to suppress the migratory and invasive capabilities of mouse embryonic fibroblasts (MEFs) following the deletion of the EIF $2 A K 2$ gene. An in vivo experimental metastasis assay revealed that kisspeptin-induced EIF2AK2 activation attenuated cancer cell migration and invasion, thus suppressing distant metastasis to the lungs. In addition, kisspeptin was demonstrated to promote the interaction between the Ras homolog gene family member A (RhoA) and EIF2AK2, resulting in the autophosphorylation of EIF2AK2. The present results suggest that kisspeptin may suppress the metastatic capabilities of cancer cells via activation of EIF2AK2. Therefore, kisspeptin treatment may be one of options in preventing cancer metastases.

\section{Materials and methods}

Cell lines and reagents. The following cell lines used in the present study were kindly provided by Professor Barat B. Aggarwal (University of Texas, Anderson Cancer Center, Houston, Texas): wild-type MEFs (MEF-EIF2AK2 $2^{+/+}$), MEFs where the EIF2AK2 gene was deleted (MEF-EIF $2 A K 2^{-/-}$), human breast SK-BR-3, prostatic PC-3 and colorectal LoVo adenocarcinoma cells. Cells were cultured in Dulbecco's modified Eagle's medium or RPMI-1640 (Cellgro; Corning Incorporated, Corning, NY, USA), supplemented with $10 \%$ fetal bovine serum (Cellgro; Corning Incorporated) and 1\% penicillin/streptomycin (Gibco; Thermo Fisher Scientific, Inc., Waltham, MA, USA) and $5 \% \mathrm{CO}_{2}$ humidified air at $37^{\circ} \mathrm{C}$. Kisspeptin (C-terminal amidated kisspeptin-10) was purchased from Tocris Bioscience (Bristol, UK). The Rho-associated protein kinase (ROCK) inhibitor Y-27632 was purchased from Selleck Chemicals (Houston, TX, USA). The EIF2AK2 inhibitor C16 was purchased from Santa Cruz Biotechnology, Inc. (sc-204200; Dallas, TX, USA).

Short interfering (si)RNA experiment. $3 \times 10^{6}$ LoVo cells were transfected with EIF2AK2 siRNA or negative control siRNA using Lipofectamine 2000 reagent (Invitrogen; Thermo Fisher Scientific, Inc.). In brief, $1 \times 10^{6}$ LoVo cells were cultured in DMEM medium without antibiotics a day before transfection. Then, $20 \mu \mathrm{l}$ of Lipofectamine 2000 reagent in $500 \mu \mathrm{l}$ of serum-free DMEM medium and $10 \mu \mathrm{l}$ of each siRNA in $500 \mu \mathrm{l}$ of serum-free DMEM medium were gently mixed and incubated for $30 \mathrm{~min}$ at room temperature, and the mixed solution was added in the cells. The cells were then incubated at $37^{\circ} \mathrm{C}$ in a $\mathrm{CO}_{2}$ incubator for $24 \mathrm{~h}$ and then used for the assays such as the migration and invasion assay. EIF2AK2 siRNA and negative control siRNA were purchased from Santa Cruz Biotechnology, Inc. These siRNA products consist of pools of three to five target-specific 19 to 25 nucleotide siRNAs designed to knock down gene expression. EIF2AK2 silencing was confirmed by western blot analysis.
Western blot analysis. A total of $3 \times 10^{6}$ cells (all cell types used in this study) were lyzed using radioimmunoprecipitation lysis buffer (20 mM Tris- $\mathrm{HCl}, \mathrm{pH} 7.5,150 \mathrm{mM} \mathrm{NaCl}, 1 \mathrm{mM}$ $\mathrm{Na}_{2}$ EDTA, 1 mM EGTA, 1\% NP-40, $1 \%$ sodium deoxycholate, $2.5 \mathrm{mM}$ sodium pyrophosphate, $1 \mathrm{mM} \beta$-glycerophosphate, $1 \mathrm{mM} \mathrm{Na} \mathrm{VO}_{4}, 1 \mu \mathrm{g} / \mathrm{ml}$ leupeptin, $1 \mathrm{mM}$ PMSF) for $30 \mathrm{~min}$ on ice and centrifuged at $20,000 \mathrm{xg}$ for $10 \mathrm{~min}$ at $4^{\circ} \mathrm{C}$. Protein concentrations were measured using Pierce BCA protein assay kit (Pierce; Thermo Fisher Scientific, Inc.), according to the manufacturer's protocol. Equal amounts of extracted protein samples $(30 \mu \mathrm{g})$ were separated by $6-12 \%$ SDS-PAGE and transferred onto polyvinylidene difluoride membranes. Subsequent to blocking with $5 \%$ milk for $1 \mathrm{~h}$ at room temperature, membranes were probed for $1 \mathrm{~h}$ at room temperature with the following primary antibodies: Anti-phosphorylated (p)-cofilin (sc-271921; 1:250; Santa Cruz Biotechnology, Inc.), anti-cofilin (sc-376476; 1:250; Santa Cruz Biotechnology, Inc.), anti-p-myosin light chain (MLC; 3674; 1:1,000; Cell Signaling Technology,Inc.,Danvers,MA,USA), anti-MLC (8505; 1:1,000; Cell Signaling Technology, Inc.), anti-RhoA (sc-166399; 1:250; Santa Cruz Biotechnology, Inc.), anti-EIF2AK2 (sc-6282; 1:250; Santa Cruz Biotechnology, Inc.) anti-p-EIF2AK2 (3075; 1:1,000; Cell Signaling Technology, Inc.) and anti- $\beta$-actin (4967; 1:1,000; Cell Signaling Technology, Inc., Danvers, MA, USA). The membranes were then incubated in secondary antibodies conjugated to horseradish peroxidase (anti-rabbit; 7074; 1:10,000, anti-mouse; 7076; 1:10,000; Cell Signaling Technology, Inc.) for $1 \mathrm{~h}$ at room temperature. Bands were detected using LumiGLO chemiluminescent reagent and peroxidase (7003; Cell Signaling Technology, Inc.). Active GTP-bound RhoA was detected by a glutathione-S-transferase (GST) pull down assay using a human Rhotekin Rho binding domain (RBD)-GST-fusion protein.

Scratch wound assay. An in vitro scratch-wound healing assay was used to evaluate cellular migration; $3 \times 10^{5}$ cells (from all cell lines tested in the present study) were cultured in 6-well plates containing DMEM medium at $37^{\circ} \mathrm{C}$ in $\mathrm{CO}_{2}$ incubator for $24 \mathrm{~h}$ and scratched when cell confluence reached about $80 \%$. A 'scratch' was created by scraping the cell culture in a straight line with a $200 \mu \mathrm{l}$ pipette tip and cells were washed with PBS three times to remove debris. The cells were then treated with kisspeptin at $100 \mathrm{nM}$ for $24 \mathrm{~h}$ at $37^{\circ} \mathrm{C}$ and then random cell migration was evaluated via counting the number of cells that had migrated in the scratched region. Experiments were performed in triplicate. Non-treated cells were used as a control in the migration assay. Cell migration was observed using a Zeiss Axiovert inverted microscope, and images were analyzed using Zen software version 3.00 (Carl Zeiss AG, Oberkochen, Germany).

Cell invasion analysis. An invasion assay was used to evaluate the invasive capabilities of cancer cells; $3 \times 10^{5}$ cells (from all cell lines tested in this study) were seeded into the upper chambers of Transwell inserts (Corning Incorporated, Corning, NY, USA) pre-coated with Matrigel (BD Biosciences, San Jose, CA, USA). The upper chambers were filled with DMEM medium supplemented with $1 \%$ serum, whereas the lower chambers were filled with DMEM medium supplemented with $10 \%$ serum as a chemoattractant. The cells in the upper chamber 
were treated with kisspeptin at $100 \mathrm{nM}$ for $24 \mathrm{~h}$. Non-treated cells were used as a control. Following incubation at $37^{\circ} \mathrm{C}$ for $24 \mathrm{~h}$, cells on the top of the membrane were carefully removed with cotton swabs. Cells that had invaded the lower membrane were fixed with $4 \%$ formaldehyde at room temperature for 5 min, stained with $1 \%$ crystal violet at room temperature for another $5 \mathrm{~min}$, and then counted. Experiments were performed in triplicate. Cell invasion was observed using a Zeiss Axiovert inverted microscope, and images were analyzed using Zen software version 3.00 (Carl Zeiss AG). A total of 4 fields were randomly selected and the invaded cells were counted.

In vivo studies. Cultured LoVo cells $\left(3 \times 10^{5}\right)$ were subcutaneously injected into the back skin of 6-week old male nude mice (Orient Bio Inc., Seongnam, Korea). A day after tumor cell injection, kisspeptin $(100 \mathrm{nM})$ alone $(\mathrm{n}=6)$, or in combination with C16 (200 nM) $(\mathrm{n}=6)$ or control (saline, $n=6)$ was subcutaneously injected every other day into the tumor site for 14 days. Tumor volume was measured every other day, according to the following formula: Tumor volume $\left(\mathrm{mm}^{3}\right)=0.5 \mathrm{x}$ (tumor length, mm) x (tumor width, $\mathrm{mm})^{2}$. Mice were sacrificed 14 days after treatment, and tumors were removed for immunohistochemical analysis. For the experimental metastasis assay, LoVo cells $\left(1 \times 10^{6}\right)$ were treated with kisspeptin $(100 \mathrm{nM})$ alone or in combination with C16 (200 nM) for $1 \mathrm{~h}$ at $37^{\circ} \mathrm{C}$, and then injected into the tail vein of the mice. A total of 2 weeks following the injection, mice were sacrificed, the lungs were isolated and metastatic foci were counted. Mice were maintained under a 12-h light/dark cycle with free access to food and water and diet at $24 \pm 1^{\circ} \mathrm{C}$ and a relative humidity of $40 \pm 5 \%$. All experimental procedures were approved by the Institutional Animal Care and Use Committee of the Kyung Hee University (Seoul, Republic of Korea).

Immunohistochemistry. Tumor xenografts were removed, cut and put on cassettes, fixed with $4 \%$ formaldehyde at room temperature for $24 \mathrm{~h}$ and embedded in paraffin wax. Tumor tissue was sectioned at $10 \mu \mathrm{m}$, deparaffinized, and antigen-retrieval performed with $8 \mathrm{M}$ Urea, $\mathrm{H}_{2} \mathrm{O}_{2}$ was used to block endogenous peroxidase. The sections were washed with TBST $(0.1 \%$ Tween-20) twice, and blocked in $10 \%$ normal serum with $1 \%$ BSA for $1 \mathrm{~h}$ at room temperature, stained with an anti-p-EIF2AK2 antibody (ab32036; Abcam, Cambridge, $\mathrm{UK}$ ) for $1 \mathrm{~h}$ at room temperature, incubated with anti-rabbit secondary antibody (ab205718; Abcam) for another $1 \mathrm{~h}$ at room temperature and then incubated with $\mathrm{DAB}$ for $2 \mathrm{~min}$ at room temperature using DAB substrate kit (ab64238; Abcam). Stained sections were observed under x20 magnification. Cell invasion was observed using a Zeiss Axiovert inverted microscope, and images were analyzed using Zen software version 3.00 (Carl Zeiss AG).

Statistical analysis. The statistical significance of the differences between groups was assessed using unpaired Student's t-test or one-way analysis of variance with a post-hoc Tukey's test using SPSS version 22 (IBM Corp., Armonk, NY, USA). All in vitro experiments were conducted at least three times. Data are expressed as the mean \pm standard deviation. $\mathrm{P}<0.05$ was considered to indicate a statistically significant difference.

\section{Results}

EIF2AK2 is involved in kisspeptin-mediated inhibition of cellular migration and invasion. To investigate whether EIF2AK2 may be involved in the mechanism underlying the inhibitory effects of kisspeptin on cellular migration, wild-type MEFs (MEF-EIF2AK2 $2^{+/+}$) and MEFs where the EIF2AK2 gene was deleted (MEF-EIF $2 A K 2^{-/}$) received a scratch wound and were subsequently treated with kisspeptin for $24 \mathrm{~h}$. EIF2AK2 expression in MEF-EIF $2 A K 2^{+/+}$, and its absence in MEF-EIF $2 A K 2^{-/}$cells were confirmed using western blot analysis (Fig. 1A). Treatment with kisspeptin was revealed to inhibit the migratory capabilities of normal MEFs; however, in MEFs where EIF2AK2 was knocked out, the inhibitory effects of kisspeptin on cellular migration were abolished (Fig. 1B). Similarly, kisspeptin failed to suppress the invasive capabilities of MEF-EIF $2 A K 2^{-/-}$cells (Fig. 1C). These results suggested that EIF2AK2 may be involved in the mechanisms underlying the inhibitory effects of kisspeptin on cellular migration and invasion.

Kisspeptin-mediated EIF2AK2 activation is crucial for the inhibition of cancer cell migration and invasion. To investigate the effects of kisspeptin on the migratory and invasive capabilities of cancer cells, SK-BR-3, PC-3 and LoVo cells were treated with kisspeptin for $24 \mathrm{~h}$ and subsequently evaluated in scratch wound and invasion assays. The present results revealed that kisspeptin significantly suppressed cancer cell migration and invasion (Fig. 2A and B).

RNA interference was used to silence the expression of EIF2AK2 in LoVo cells (Fig. 2C). Following EIF2AK2 knockdown, the inhibitory effects of kisspeptin on cancer cell migration and invasion were abolished (Fig. 2D and E). The results of the present study suggested that kisspeptin may exert its suppressive effects on the metastatic capabilities of cancer cells via an EIF2AK2-mediated pathway.

Kisspeptin-mediated EIF $2 A K 2$ phosphorylation requires RhoA activation. Kisspeptin has been reported to modulate the RhoA intracellular signaling pathway $(8,12,27)$. In LoVo and SK-BR-3 cells, a GST pull-down assay demonstrated that kisspeptin activated RhoA (Fig. 3A). Western blot analysis revealed that treatment with kisspeptin increased the phosphorylation of the downstream mediators of RhoA, MLC and cofilin (Fig. 3A). Notably, the inhibition of EIF2AK2 with C16 (100 nM) did not appear to prevent the activation of RhoA in LoVo cells (Fig. 3B). However, the inhibition of the downstream effector of RhoA ROCK with Y-27632 (100 nM) appeared to prevent the phosphorylation of EIF2AK2 in LoVo cells (Fig. 3C). These results suggested that RhoA-mediated signaling may be implicated in the mechanisms underlying the kisspeptin-induced phosphorylation of EIF2AK2.

Kisspeptin-induced EIF2AK2 activation inhibits cancer metastasis in vivo. Kisspeptin has been reported to suppress cancer metastasis $(6,7)$. The aforementioned results suggested that kisspeptin-induced EIF2AK2 activation may be involved in the inhibitory effects on cancer cell motility. Therefore, the implication of EIF2AK2 in the mechanism of action of 


$$
\text { A }
$$

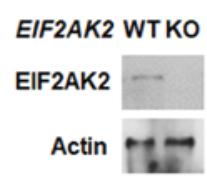

B
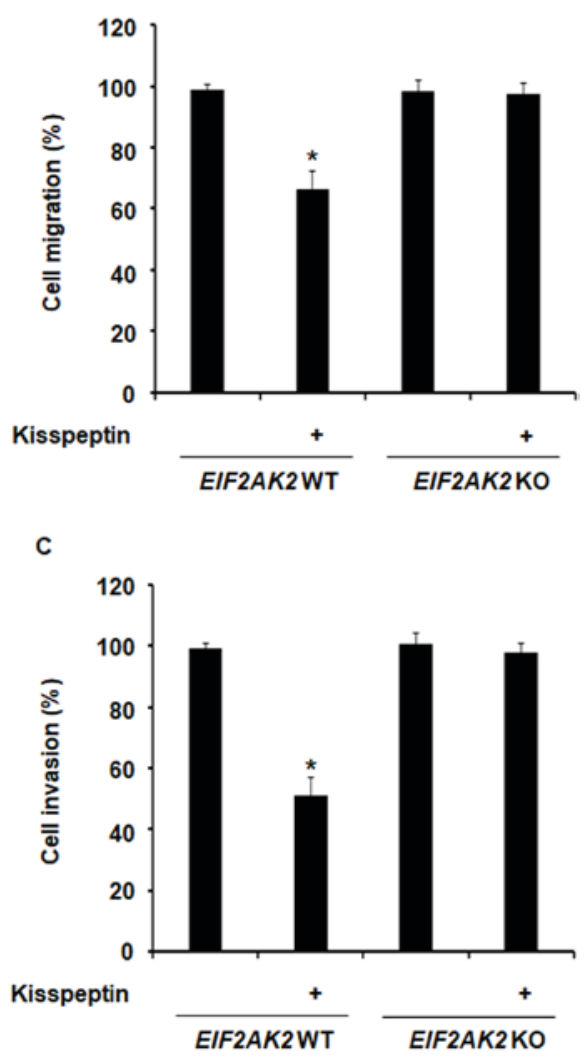

Figure 1. EIF2AK2 is involved in kisspeptin-mediated inhibition of cellular migration and invasion. (A) Western blot analysis was used to assess the protein expression levels of EIF2AK2 in wild-type MEFs (MEF-EIF2AK2 $2^{+/+}$) and MEFs where the EIF2AK2 gene was knocked out (MEF-EIF2AK2 $2^{-1}$ ). (B) A scratch wound assay was used to evaluate the migratory capabilities of MEFs. A scratch wound was inflicted and cells were treated with kisspeptin $(100 \mathrm{nM})$. The number of cells migrated into the wound area was counted. (C) A Matrigel invasion assay was used to evaluate the invasive capabilities of MEFs. Kisspeptin (100 nM) was added to the upper chambers and the number of invaded cells was counted. Data are expressed as the mean \pm standard deviation of 3 independent experiments. ${ }^{*} \mathrm{P}<0.05$ vs. control. EIF2AK2, eukaryotic translation initiation factor $2 \alpha$ kinase 2; MEF, mouse embryonic fibroblast; WT, wild-type; KO, knock-out.

kisspeptin was investigated in cancer metastasis in vivo. LoVo-derived tumor xenografts were implanted into nude mice, and kisspeptin administration was revealed to suppress tumor growth (Fig. 4A). Notably, the inhibition of EIF2AK2 following $\mathrm{C} 16$ co-administration appeared to abolish the inhibitory effects of kisspeptin in vivo (Fig. 4A). Similarly, following treatment with kisspeptin, phosphorylation levels of EIF2AK2 in xenografted tumor tissue appeared to be increased, whereas C16 effectively prevented EIF2AK2 phosphorylation (Fig. 4B).

An experimental metastasis assay was used to investigate the effects of kisspeptin on the metastatic capabilities of cancer cells in vivo. The present results demonstrated that kisspeptin prevented the development of LoVo-derived lung metastases
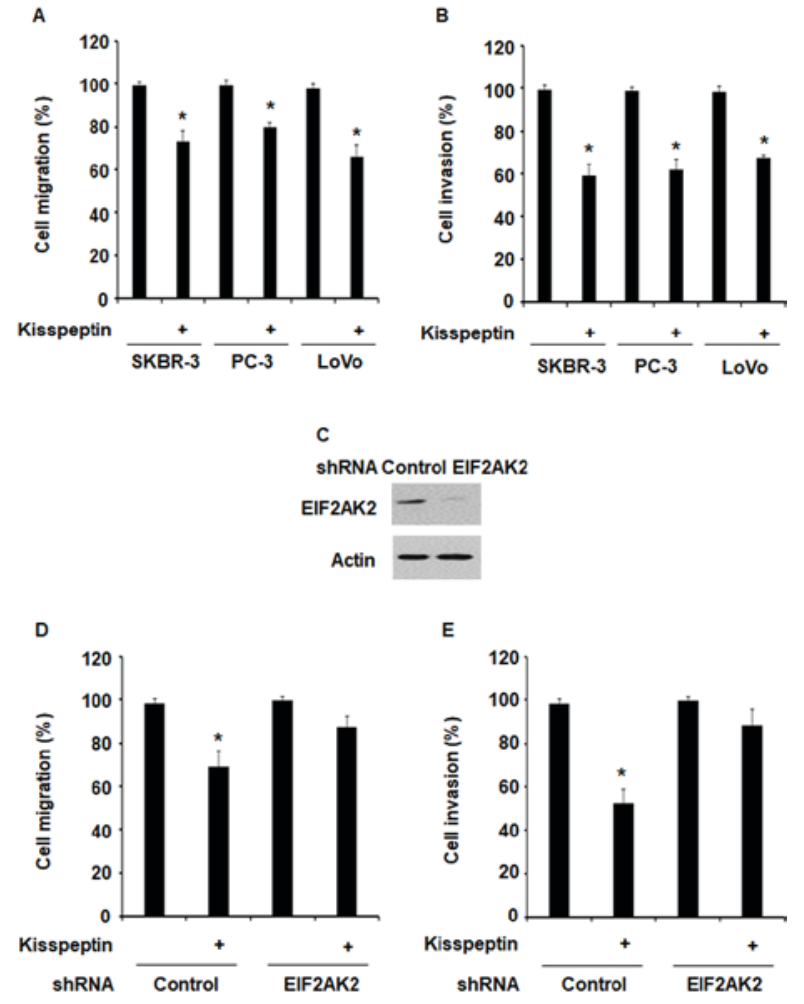

Figure 2. Kisspeptin-mediated EIF2AK2 activation is involved in the inhibition of cancer cell migration and invasion. Human SK-BR-3 breast, PC-3 prostatic and LoVo colorectal adenocarcinoma cells were treated with kisspeptin (100 $\mathrm{nM}$ ) and their (A) migratory and (B) invasive capabilities were assessed. (C) RNA interference was used to silence the expression of EIF2AK2 in LoVo cells. The (D) migratory and (E) invasive capabilities of LoVo cells were assessed following knockdown of EIF2AK2 expression. Data are expressed as the mean \pm standard deviation of 3 independent experiments. ${ }^{*} \mathrm{P}<0.05$ vs. control. EIF2AK2, eukaryotic translation initiation factor $2 \alpha$ kinase 2 ; sh, short hairpin.

in nude mice, whereas EIF2AK2 inhibition with $\mathrm{C} 16$ abolished the inhibitory effects of kisspeptin on the metastatic capabilities of LoVo cells (Fig. 4C). These results suggested that kisspeptin may inhibit cancer growth and metastasis in vivo through the activation of EIF2AK2-mediated signaling pathways.

\section{Discussion}

Kisspeptin is a protein encoded by the KISS1 gene, which has been reported to suppress the metastatic capabilities of various types of cancer cells $(6,7)$, and KISS1 expression levels have been revealed to be downregulated in metastatic compared with non-metastatic cancer tissue (28). However, those findings inversely indicate that KISSI expression level is higher in tumor tissues than in normal tissues. Conversely, the G-protein coupled receptor for kisspeptin, GPR54, has been implicated in breast cancer development (27). These contradictory results suggest that the effects of kisspeptin during cancer development and metastasis may differ across various types of cancer cells (5). Kisspeptin-mediated intracellular signaling has been reported to serve diverse roles in physiological and pathological conditions; however, the implication of kisspeptin-mediated pathways in the regulation of cancer cell migration and invasion has yet to be elucidated. The results of the present study 


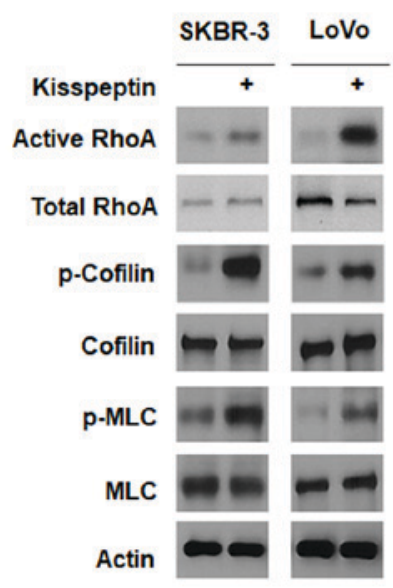

B

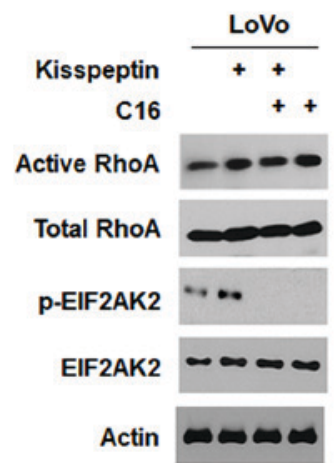

C

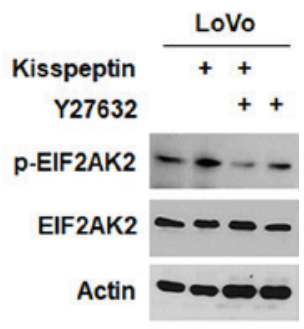

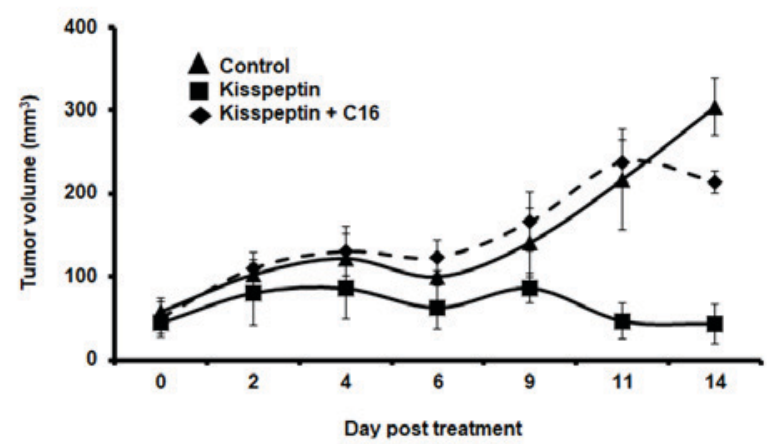

B
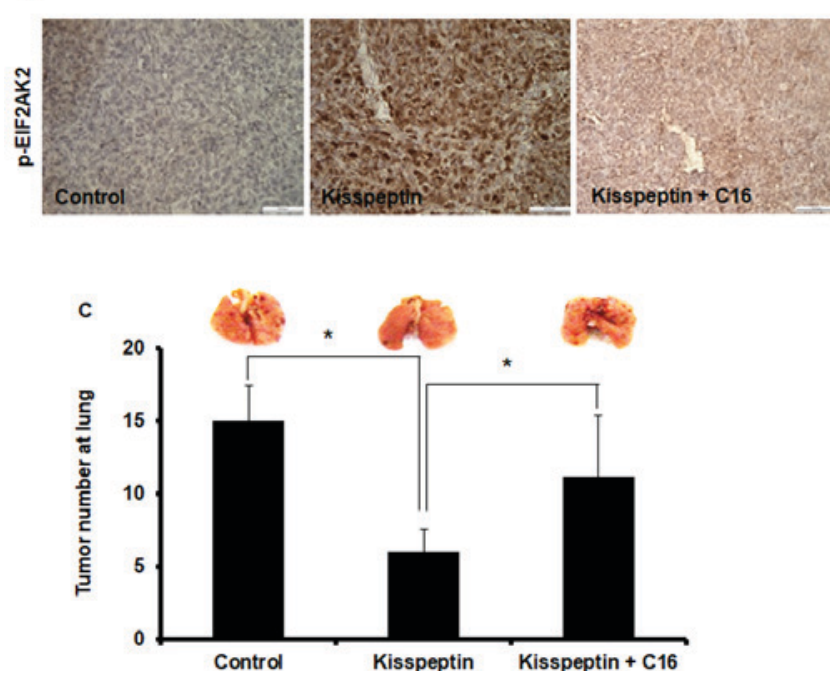

Figure 4. Kisspeptin inhibits tumor growth and metastasis in vivo. (A) Mice were subcutaneously injected with human LoVo colorectal adenocarcinoma cells to develop a tumor xenograft model. Mice were then treated with kisspeptin alone or in combination with the EIF2AK2 inhibitor C16 and tumor growth was evaluated for 14 days. (B) Tumor xenograft tissue samples were obtained from mice and p-EIF2AK2 protein expression was examined using immunohistochemistry. Photomicrographs were captured under x20 magnification. Scale bars, $100 \mu \mathrm{m}$. (C) The effects of kisspeptin on the metastatic capabilities of cancer cells were assessed in vivo. LoVo cells were injected into the tail vein of nude mice and pulmonary metastasis was examined 2 weeks following the injection. Lung images are shown on the top of bars in the graph. ${ }^{*} \mathrm{P}<0.05$. EIF2AK2, eukaryotic translation initiation factor $2 \alpha$ kinase 2; p-, phosphorylated.

Kisspeptin has been reported to activate RhoA via a GPR54/ $\mathrm{G}_{\mathrm{q} / 11} / \mathrm{p} 63$ RhoA-specific guanine nucleotide exchange factor (p63RhoGEF)-mediated signaling pathway (27). The present study suggested that RhoA may be required for the kisspeptin-induced activation of EIF2AK2. Therefore, it may be hypothesized that the signaling pathway underlying the effects of kisspeptin may be represented by the following scheme: GPR54/G $\mathrm{q}_{\mathrm{q} / 1} / \mathrm{p} 63 \mathrm{RhoGEF} / \mathrm{RhoA} / \mathrm{EIF} 2 \mathrm{AK} 2$.

The results of the present study suggested that autophagy may be linked to cytoskeletal dynamics (29), since the roles of EIF2AK2 in the regulation of autophagy have been previously demonstrated (30). However, the effects of kisspeptin in the regulation of autophagy have yet to be elucidated, and further studies are required to investigate whether kisspeptin-mediated EIF2AK2 activation may modulate autophagy, as well as the association between autophagy and cytoskeletal dynamics. Vesicle trafficking has been associated with cytoskeletal dynamics (31). Therefore, it may be hypothesized that the pathways. 
trafficking of autophagosomes by cytoskeletal components serves a role in cancer cell motility, as well as in their metastatic capabilities. However, further studies are required to investigate the putative relationship between autophagy and cell motility. In conclusion, the present study suggested that kisspeptin may regulate the metastatic capabilities of cancer cells via EIF2AK2-associated signaling pathways in vitro and in vivo. The present study, therefore, may improve our knowledge of the role of kisspeptin as a metastasis suppressor and address its pharmaceutical application in cancer treatment.

\section{Acknowledgements}

The present study was supported by the Basic Science Research Program through the National Research Foundation of Korea funded by the Ministry of Science, ICT and Future Planning (grant no. NRF-2014R1A1A1035831).

\section{References}

1. Oakley AE, Clifton DK and Steiner RA: Kisspeptin signaling in the brain. Endocr Rev 30: 713-743, 2009.

2. Colledge WH, Doran J and Mei H: Model systems for studying kisspeptin signalling: Mice and cells. Adv Exp Med Biol 784: 481-503, 2013.

3. Silveira LG, Latronico AC and Seminara SB: Kisspeptin and clinical disorders. Adv Exp Med Biol 784: 187-199, 2013.

4. Castellano JM and Tena-Sempere M: Metabolic regulation of kisspeptin. Adv Exp Med Biol 784: 363-383, 2013.

5. Cho SG, Li D, Tan K, Siwko SK and Liu M: KiSS1 and its G-protein-coupled receptor GPR54 in cancer development and metastasis. Cancer Metastasis Rev 31: 585-591, 2012.

6. Lee JH and Welch DR: Suppression of metastasis in human breast carcinoma MDA-MB-435 cells after transfection with the metastasis suppressor gene, KiSS-1. Cancer Res 57: 2384-2387, 1997.

7. Ohtaki T, Shintani Y, Honda S, Matsumoto H, Hori A, Kanehashi K, Terao Y, Kumano S, Takatsu Y, Masuda Y, et al: Metastasis suppressor gene KiSS-1 encodes peptide ligand of a G-protein-coupled receptor. Nature 411: 613-617, 2001.

8. Navenot JM, Fujii N and Peiper SC: Activation of Rho and Rho-associated kinase by GPR54 and KiSS1 metastasis suppressor gene product induces changes of cell morphology and contributes to apoptosis. Mol Pharmacol 75: 1300-1306, 2009.

9. Tan K, Cho SG, Luo W, Yi T, Wu X, Siwko S, Liu M and Yuan W: KiSS1-induced GPR54 signaling inhibits breast cancer cell migration and epithelial-mesenchymal transition via protein kinase D1. Curr Mol Med 14: 652-662, 2014.

10. Jiang Y, Berk M, Singh LS, Tan H, Yin L, Powell CT and Xu Y: KiSS1 suppresses metastasis in human ovarian cancer via inhibition of protein kinase C alpha. Clin Exp Metastasis 22: 369-376, 2005.

11. Roseweir AK, Katz AA and Millar RP: Kisspeptin-10 inhibits cell migration in vitro via a receptor-GSK3 beta-FAK feedback loop in HTR8SVneo cells. Placenta 33: 408-415, 2012.

12. Cho SG, Li D, Stafford LJ, Luo J, Rodriguez-Villanueva M, Wang Y and Liu M: KiSS1 suppresses TNFalpha-induced breast cancer cell invasion via an inhibition of RhoA-mediated NF-kappaB activation. J Cell Biochem 107: 1139-1149, 2009.

13. Stafford LJ, Xia C, Ma W, Cai Y and Liu M: Identification and characterization of mouse metastasis-suppressor KiSS1 and its G-protein-coupled receptor. Cancer Res 62: 5399-5404, 2002.
14. Williams BR: PKR; a sentinel kinase for cellular stress. Oncogene 18: 6112-6120, 1999.

15. Meurs EF, Galabru J, Barber GN, Katze MG and Hovanessian AG Tumor suppressor function of the interferon-induced double-stranded RNA-activated protein kinase. Proc Natl Acad Sci USA 90: 232-236, 1993.

16. Hii SI, Hardy L, Crough T, Payne EJ, Grimmett K, Gill D and McMillan NA: Loss of PKR activity in chronic lymphocytic leukemia. Int J Cancer 109: 329-335, 2004.

17. Shir A and Levitzki A: Inhibition of glioma growth by tumor-specific activation of double-stranded RNA-dependent protein kinase PKR. Nat Biotechnol 20: 895-900, 2002.

18. Watanabe MA, Rodrigues Souza L, Murad JM and De Lucca FL: Antitumor activity induced by regulatory RNA: Possible role of RNA-dependent protein kinase and nuclear factor-kappaB. Eur J Pharmacol 465: 205-210, 2003.

19. Kim SH, Gunnery S, Choe JK and Mathews MB: Neoplastic progression in melanoma and colon cancer is associated with increased expression and activity of the interferon-inducible protein kinase, PKR. Oncogene 21: 8741-8748, 2002.

20. Kim SH, Forman AP, Mathews MB and Gunnery S: Human breast cancer cells contain elevated levels and activity of the protein kinase, PKR. Oncogene 19: 3086-3094, 2000.

21. Haines GK, Cajulis R, Hayden R, Duda R, Talamonti M and Radosevich JA: Expression of the double-stranded RNA-dependent protein kinase (p68) in human breast tissues. Tumour Biol 17: 5-12, 1996.

22. Savinova O, Joshi B and Jagus R: Abnormal levels and minimal activity of the dsRNA-activated protein kinase, PKR, in breast carcinoma cells. Int J Biochem Cell Biol 31: 175-189, 1999.

23. Xu M, Chen G, Wang S, Liao M, Frank JA, Bower KA, Zhang Z, Shi $\mathrm{X}$ and Luo J: Double-stranded RNA-dependent protein kinase regulates the motility of breast cancer cells. PLoS One 7: e47721, 2012.

24. Yuan X, Wang W, Li J, Zheng P, Dong P, Chen L, Zhou Y, Xie G, Xu D, Liu Y and Shen L: Gelsolin suppresses gastric cancer metastasis through inhibition of PKR-p38 signaling. Oncotarget 7: 53459-53470, 2016.

25. Wang X, Dong JH, Zhang WZ, Leng JJ, Cai SW, Chen MY and Yang X: Double stranded RNA-dependent protein kinase promotes the tumorigenic phenotype in HepG2 hepatocellular carcinoma cells by activating STAT3. Oncol Lett 8: 2762-2768, 2014.

26. Irving AT, Wang D, Vasilevski O, Latchoumanin O, Kozer N, Clayton AH, Szczepny A, Morimoto H, Xu D, Williams BR and Sadler AJ: Regulation of actin dynamics by protein kinase $\mathrm{R}$ control of gelsolin enforces basal innate immune defense. Immunity 36: 795-806, 2012

27. Cho SG, Wang Y, Rodriguez M, Tan K, Zhang W, Luo J, Li D and Liu M: Haploinsufficiency in the prometastasis Kiss1 receptor Gpr54 delays breast tumor initiation, progression, and lung metastasis. Cancer Res 71: 6535-6546, 2011.

28. Shengbing Z, Feng LJ, Bin W, Lingyun G and Aimin $\mathrm{H}$ : Expression of KiSS-1 gene and its role in invasion and metastasis of human hepatocellular carcinoma. Anat Rec (Hoboken) 292: 1128-1134, 2009.

29. Caino MC, Chae YC, Vaira V, Ferrero S, Nosotti M, Martin NM, Weeraratna A, O'Connell M, Jernigan D, Fatatis A, et al: Metabolic stress regulates cytoskeletal dynamics and metastasis of cancer cells. J Clin Invest 123: 2907-2920, 2013.

30. Pietrocola F, Izzo V, Niso-Santano M, Vacchelli E, Galluzzi L, Maiuri MC and Kroemer G: Regulation of autophagy by stress-responsive transcription factors. Semin Cancer Biol 23: 310-322, 2013.

31. Mackeh R, Perdiz D, Lorin S, Codogno P and Poüs C: Autophagy and microtubules-new story, old players. J Cell Sci 126: 1071-1080, 2013. 